\title{
SCHWANNOMA DO FORAME MAGNO: REVISÃO E RELATO DE CASO*
}

\author{
Marcelo Souto Nacif ${ }^{1}$, Stella Caiado ${ }^{2}$, Ricardo Andrade Fernandes de Mello ${ }^{3}$, \\ Gustavo Federico Jauregui ${ }^{4}$, Flávio do Amaral Campos ${ }^{5}$, Nidia Di Paula Silva Oliveira ${ }^{6}$, \\ Walter Teixeira de Paula Neto ${ }^{7}$, Alair Augusto Sarmet Moreira Damas dos Santos ${ }^{8}$
}

Resumo Os autores relatam um volumoso schwannoma cervical de apresentação incomum, numa paciente do sexo feminino, 53 anos de idade, que em fevereiro de 2002 apresentou queixa de fraqueza progressiva, iniciada há três anos, com dificuldade de mobilização das pernas e braços. Antes da cirurgia foi realizada ressonância magnética da região cervical. No exame físico apresentava postura com aumento da base de sustentação, marcha instável e paraparética, além de diminuição da força nos quatro membros, maior à esquerda, associada a dispnéia. Na ressonância magnética cervical evidenciou-se lesão expansiva extramedular de limites bem definidos, na altura de C1 e C2. Na cirurgia, o volumoso tumor encontrava-se ântero-lateralmente à medula, aderido à raiz esquerda de $\mathrm{C} 1$, estendendo-se superiormente através do forame magno, com localização extradural. 0 diagnóstico de schwannoma foi confirmado pela histopatologia. A paciente evoluiu satisfatoriamente, com melhora progressiva da hemiparesia e hemiparestesia esquerda. A ressonância magnética possibilita a detecção e avaliação da lesão, porém o diagnóstico definitivo só é feito com o exame histopatológico. Dessa forma, o diagnóstico precoce através da ressonância magnética e a exérese cirúrgica tornam-se a melhor forma de abordagem, com bom prognóstico.

Unitermos: Schwannoma; Sistema nervoso central; Forame magno.

Abstract Foramen magnum schwannoma: review of the literature and report of a case.

The authors report an unusual presentation of a voluminous neck schwannoma in a 53-year-old female that presented with a three-year history of progressive weakness associated with impaired movement of the limbs. Neurological examination revealed postural instability, unstable and paraparetic gait, tetraparesis and dyspnea. A preoperative magnetic resonance imaging of the neck revealed an expansive, extradural, wellcircumscribed lesion, with soft-tissue attenuation, at the level of C1-C2 vertebral bodies. During surgery, the tumor was found to be extradural, lateral to the cervical spinal cord, attached to the $\mathrm{C} 1$ left nerve root and extending upwards through the foramen magnum. Histopathological analysis of the resected specimen confirmed the diagnosis of schwannoma. The patient showed a favorable outcome with progressive improvement of the symptoms. Magnetic resonance imaging proved to be valuable in the detection and evaluation of the lesion, although the definite diagnosis was achieved only after histopathological studies. We concluded that magnetic resonance imaging for early diagnosis and prompt surgical resection seems to be the best approach to achieve good prognosis.

Key words: Schwannoma; Central nervous system; Foramen magnum.

* Trabalho realizado na Faculdade de Medicina de Teresópolis, Centro de Ciências Biomédicas, Fundação Educacional Serra dos Órgãos (FMT-CCBM - FESO), Teresópolis, RJ, e no Instituto de Pós-Graduação Médica Carlos Chagas (IPGMCC), Niterói, RJ.

1. Professor Auxiliar da Disciplina de Radiologia da FMT-CCBM - FESO, Mestrando do Departamento de Radiologia da Universidade Federal do Rio de Janeiro (UFRJ), Membro da Comissão de Residência Médica da Sociedade Brasileira de Radiologia (SBR), Pós-graduado pelo IPGMCC.

2. Médica Diplomada pela FMT-CCBM - FESO.

3. Mestre em Radiologia pelo Departamento de Radiologia da UFRJ, Pós-graduado pelo IPGMCC.

4. Pós-graduado pelo IPGMCC, "Staff" do Hospital Geral de Bonsucesso, VOT Imagem e Hospital São Lucas.

5. Ex-Monitor da Disciplina de Radiologia da FMT-CCBM FESO, Residente de Radiologia da Universidade Federal de São Paulo (Unifesp).

6. Professora Convidada da Disciplina de Radiologia da FMTCCBM - FESO, Residente do Hospital Casa de Portugal.

7. Ex-Monitor Bolsista da Disciplina de Radiologia da FMTCCBM - FESO.

8. Professor Titular do Curso de Pós-Graduação em Radiologia do IPGMCC, Professor Adjunto e Chefe do Serviço de Radiologia do Hospital Universitário Antônio Pedro da Universidade Federal Fluminense (HUAP-UFF), Chefe dos Centros de Diagnós-

\section{INTRODUÇÃO}

Os tumores da bainha nervosa são tumores que se originam predominantemente das células de Schwann, que derivam da crista neural, apresentando, portanto, origem neuroectodérmica. Na região cervical são raros, sendo a maioria benignos e representados pelos schwannomas (neurinomas). Foram descritos pela primeira vez

tico por Imagem dos Hospitais de Clínicas de Niterói e da VOT Imagem.

Endereço para correspondência: Dr. Marcelo Souto Nacif. Rua Álvares de Azevedo, 130, ap. 704, bloco B, Icaraí. Niterói, RJ, 24220-021. E-mail: marcelonacif30@hotmail.com e msnacif @yahoo.com.br

Recebido para publicação em 27/5/2003. Aceito, após revisão, em 8/12/2003. por Verocay em 1910 e acometem indivíduos geralmente entre 40 e 50 anos de idade, sendo mais freqüentes em mulheres.

Os schwannomas cervicais podem ser divididos em mediais e laterais ${ }^{(\mathbf{1})}$. Os mediais se originam dos nervos glossofaríngeo, vago, acessório ou hipoglosso, ou da cadeia simpática cervical. Os laterais se originam do tronco cervical, plexo cervical ou plexo braquial. Os schwannomas laterais que se originam dos nervos espinhais podem se estender através do forame espinhal para o canal espinhal, levando à formação do tumor em forma de "halteres", com componentes intra e extra-espinhal. Tumores originários de alguns nervos cranianos também podem apresentar aspecto em 
"halteres" em relação à base do crânio ${ }^{(2)}$. Podem estar associados à doença de von Recklinghausen $^{(2,3)}$. Estatisticamente, $15 \%$ dos tumores do sistema nervoso central (SNC) são espinhais; destes, $45 \%$ são benignos ( $43 \%$ a $58 \%$ neurinomas) e proporções semelhantes para os meningiomas, o grande diagnóstico diferencial. Os tumores presentes no forame magno representam de $1 \%$ a $3 \%$ dos tumores do SNC, sendo $34 \%$ destes de neurinomas ${ }^{(4-7)}$.

\section{RELATO DO CASO}

Paciente de 53 anos de idade, sexo feminino, branca, natural e residente em Magé, RJ. Há três anos procurou assistência médica com queixa de fraqueza progressiva nas pernas e braços, dificuldade progressiva para subir escadas, entrar no ônibus e estender roupas no varal. Não apresentava déficits focais, apenas dificuldade na realização de médios e grandes esforços. Recebendo diagnóstico inicial de esclerose múltipla, fez uso de polivitamínicos e sintomáticos, sem melhora.

Em fevereiro de 2002, a paciente apresentou dispnéia aos médios esforços e piora da força motora, com dificuldade para deambular, necessitando de ajuda, porém sem dor. Apresentava também diminuição da força de preensão, principalmente na mão esquerda. A paciente é hipertensa há dez anos e faz uso contínuo de captopril. Negava outras doenças.
No exame físico, estava lúcida, orientada e cooperativa. Apresentava postura típica de aumento da base de sustentação, marcha instável, paraparética, precisando de ajuda para deambular. Apresentava diminuição de força dos quatro membros, sendo maior à esquerda, principalmente em membros inferiores. Possuía discreta hiper-reflexia, sem predominância de lados, principalmente patelares, porém sem sinais de piramidalismo. Apresentava movimentos dismétricos às provas dedo-nariz-dedo

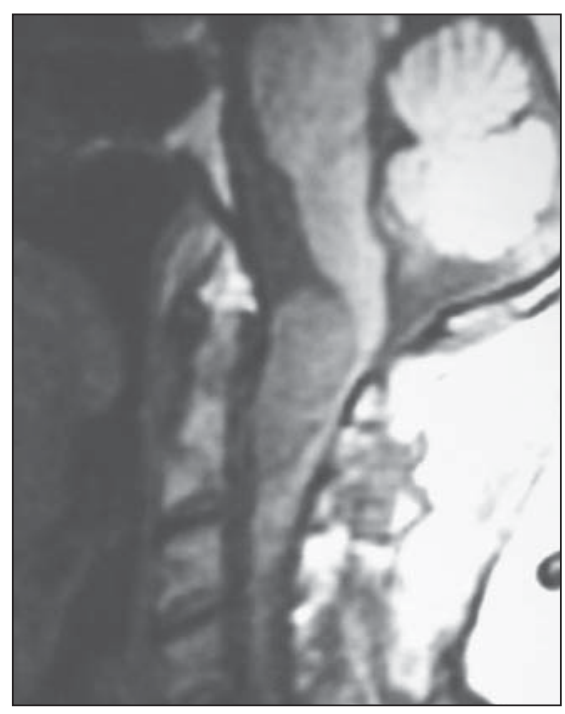

A

Figura 1. Ressonância magnética da coluna cervical no plano sagital, antes (A) e após (B) a injeção endovenosa do meio de contraste, em que se evidencia lesão expansiva de limites definidos e contornos ligeiramente lobulados, de localização intradural e extramedular na coluna cervical superior, na altura de C1 e C2. A lesão determina acentuada compressão medular e apresenta realce heterogêneo após a injeção do meio de contraste.

e calcanhar-crista tibial, com piora da dismetria à esquerda. Sensibilidades tátil, térmica e dolorosa presentes e simétricas. Sem alterações de pares cranianos. Esfíncteres competentes.

Foi realizada ressonância magnética (RM) de coluna cervical (Figuras 1 e 2), que evidenciava lesão expansiva de limites definidos e contornos algo lobulados, de localização intradural e extramedular na coluna cervical superior, na altura de $\mathrm{C} 1$ e C2. A lesão determinava acentuada com-

B

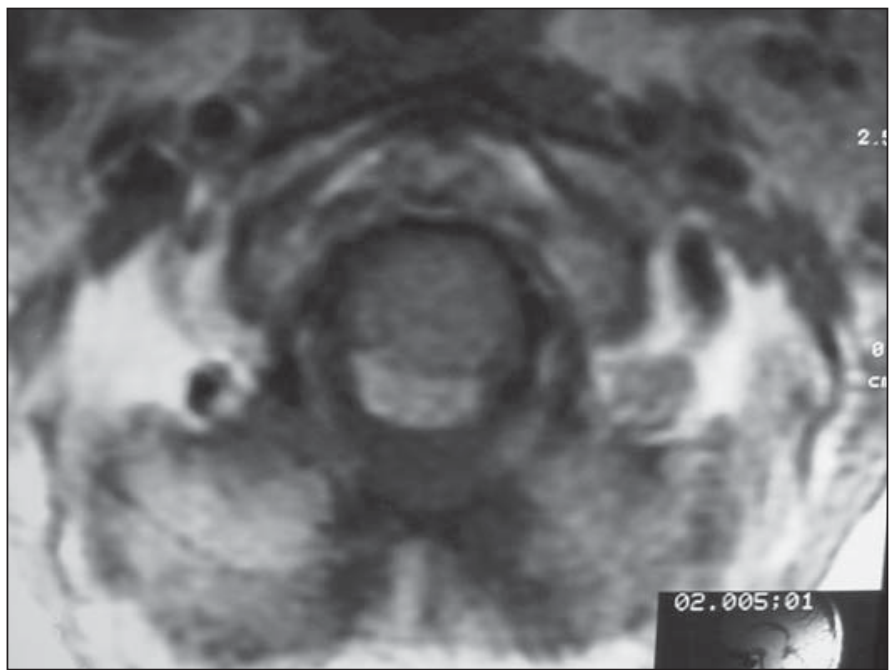

A

Figura 2. Ressonância magnética da coluna cervical ponderada em T1 sem contraste, no plano axial, em que se observa a importante compressão medular (A) e a extensão lateral da lesão através do forame neural esquerdo, que se encontra alargado (B).

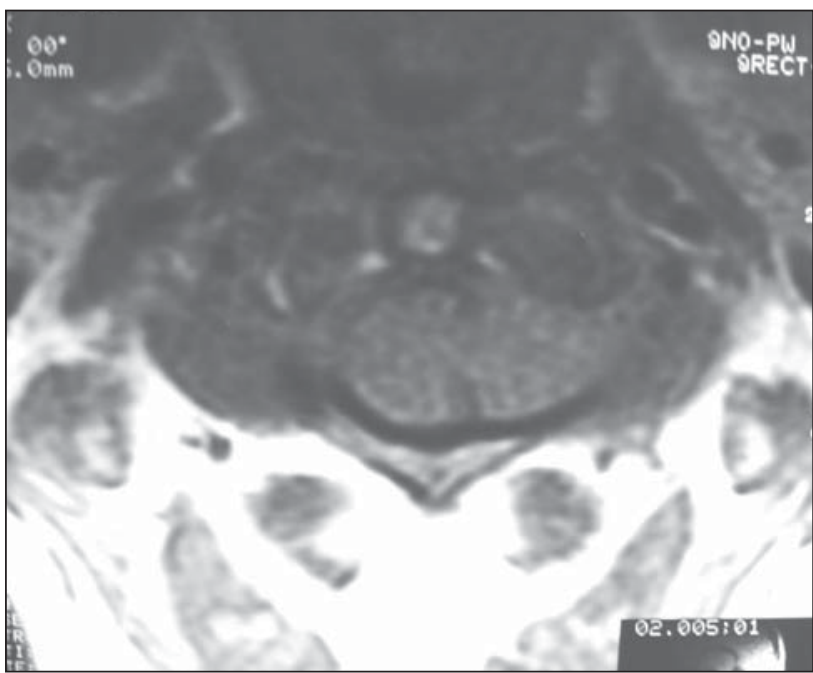

B

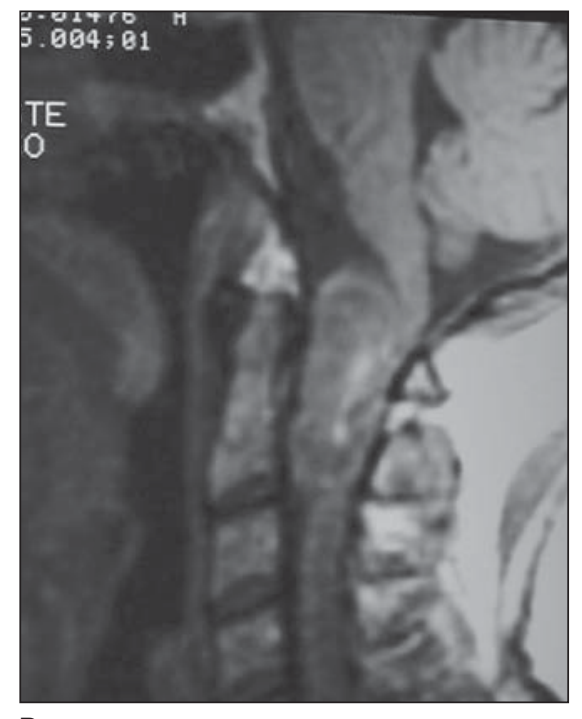


pressão medular e mostrava realce heterogêneo após a injeção do meio de contraste. Observava-se extensão lateral da lesão através do forame neural esquerdo correspondente, que se encontrava alargado.

A paciente foi submetida à cirurgia de ressecção da massa tumoral. A incisão foi de $15 \mathrm{~cm}$, na linha média posterior, indo do ínion occipital até a apófise espinhosa de C7. Foi realizada laminectomia de C1 e C2 e craniectomia da base do occipital para alargamento do forame magno. Visualizaram-se a medula e o tumor à esquerda fortemente aderido à dura e englobando a raiz de $\mathrm{C} 1$. A exérese tumoral causou grande sangramento do plexo venoso epidural (Figura 3).

A peça foi enviada para estudo histopatológico, que apresentou: células fusiformes com núcleos em forma de charutos, compactadas em feixes de várias direções; ao corte longitudinal, núcleos vizinhos, lado a lado, intercalados por espaços de menor celularidade que se repetiam; tecidos Antoni A e Antoni B, além de degenerações com formações císticas, calcificações e hemorragia. Imuno-histoquímica: proteína S-100 (Figura 4).
O pós-operatório foi sem complicações, com melhora progressiva dos sintomas. A paciente recebeu alta hospitalar no quinto dia pós-operatório, tendo ficado os três primeiros dias na unidade de tratamento intensivo. Vem evoluindo com melhora da hemiparesia e hemiparestesia esquerda.

\section{DISCUSSÃO}

Os schwannomas, quando se estendem para a região intra-espinhal, podem causar sintomas compressivos e aumento do forame espinhal ${ }^{(3,7,8)}$. Quando no forame

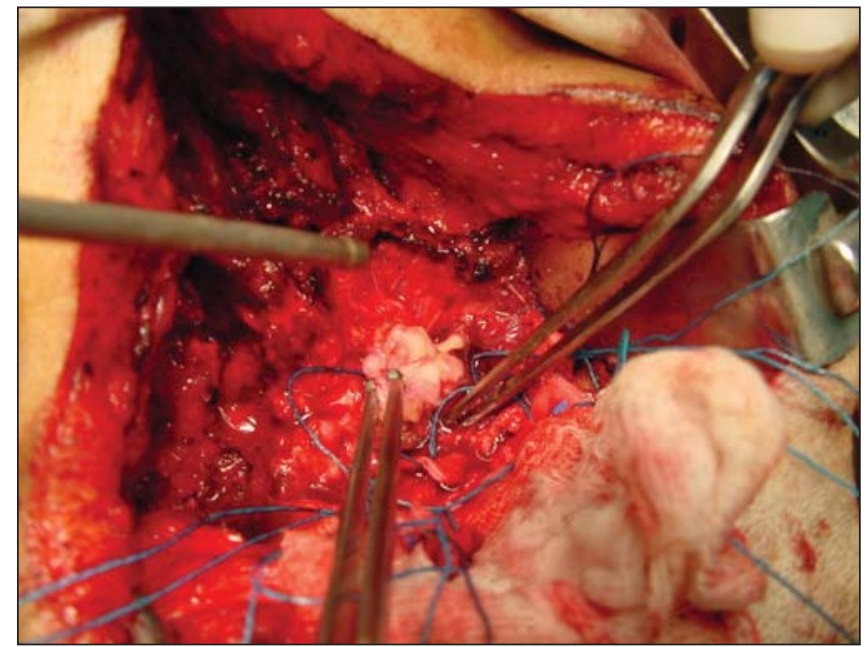

A

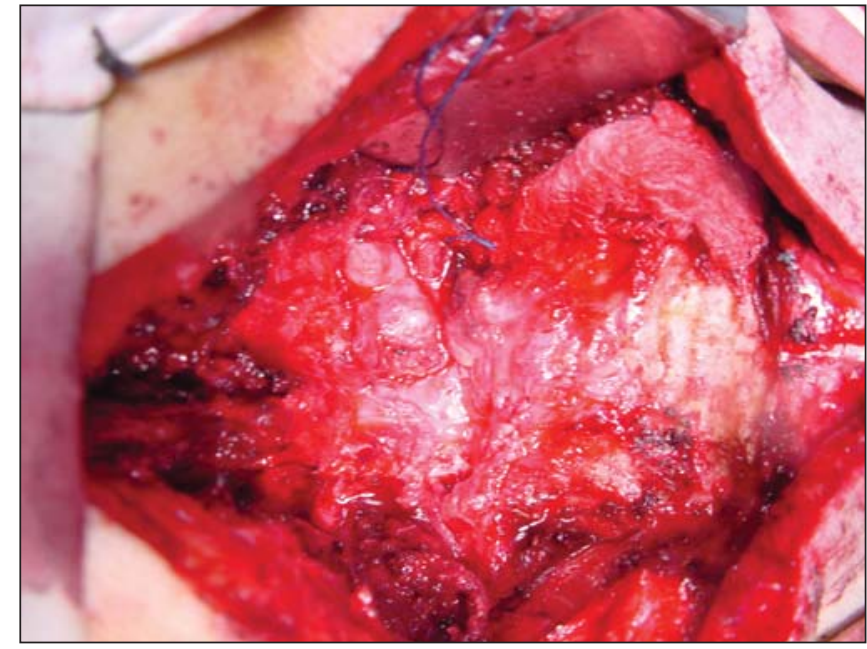

B

Figura 3. Fotografias da cirurgia de ressecção da massa tumoral à qual a paciente foi submetida. A incisão foi de $15 \mathrm{~cm}$, na linha média posterior, indo do ínion occipital até a apófise espinhosa de C7. Foi realizada laminectomia de C1 e C2 e craniectomia da base do occipital para alargamento do forame magno.Visualizaramse a medula e o tumor à esquerda fortemente aderido à dura e englobando a raiz de C1.

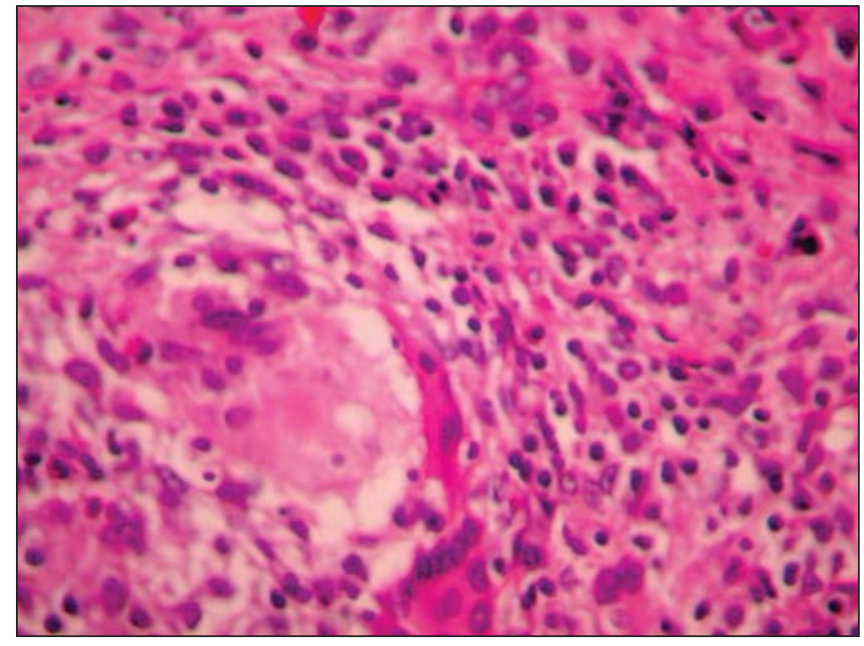

A

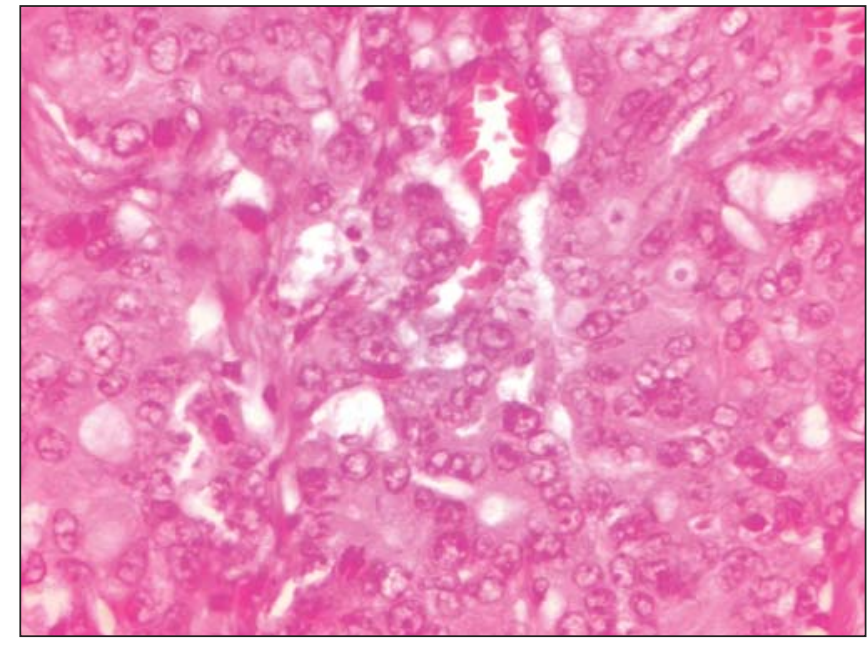

B

Figura 4. Cortes histológicos corados pela hematoxilina-eosina, em pequeno (A) e grande aumento (B), que mostram células fusiformes compactadas em feixes de várias direções, com núcleos vizinhos dispostos lado a lado, intercalados por espaços de menor celularidade que se repetem: tecidos Antoni A e Antoni B, além de degenerações com formações císticas, calcificações e hemorragia. 
magno, podem provocar sintomas por compressão bulbar, podendo levar à morte se não diagnosticado.

Macroscopicamente, são tumores encapsulados, aderidos ou envoltos por um nervo ${ }^{(\mathbf{2 , 5 , 9 )}}$. Geralmente apresentam alterações degenerativas, tanto císticas quanto necro-hemorrágicas ${ }^{(6,7,9)}$.

Os schwannomas se originam e crescem na periferia dos nervos, os quais são comprimidos e deslocados lateralmente pela massa tumoral ${ }^{(\mathbf{1 0})}$. Os nervos podem ser identificados ao longo do tumor, passando lateralmente a este ${ }^{(\mathbf{1 0 , 1 1})}$.

Microscopicamente, apresentam aspecto característico, com predomínio de células de Schwann e ausência de tecido nervoso ou fibras colágenas. Observa-se mistura de dois padrões histológicos, os tecidos Antoni A e Antoni $\mathrm{B}^{(4,5,10)}$. As áreas de tecido Antoni A têm arranjo celular compacto, enquanto as áreas de tecido Antoni B apresentam uma textura esparsa, mucinosa, com estroma formando malha de microcistos $^{(2,5,6)}$.

Os schwannomas comprimem e empurram as fibras nervosas, que são encontradas apenas na periferia do tumor, comprimidas abaixo da cápsula ${ }^{(\mathbf{1 , 2 , 7 , 8 )}}$.

$\mathrm{Na}$ TC esses tumores aparecem como massas hipodensas, heterogêneas e bem circunscritas. O padrão heterogêneo ocorre devido à presença de áreas alternadas de tecidos Antoni A e Antoni B e áreas de degeneração cística $^{(\mathbf{7 , 9 - 1 1 )}}$. Após a administra- ção endovenosa do meio de contraste, a lesão costuma apresentar realce intenso homogêneo ou heterogêneo, com zonas persistentemente hipodensas causadas por necrose ou degeneração cística e, ocasionalmente, realce anelar ${ }^{(3-5)}$. Na RM apresentam intensidade de sinal baixo a intermediário nas imagens ponderadas em T1 e intensidade de sinal alto e heterogêneo em $\mathrm{T} 2$, que correspondem a áreas alternadas de celularidade Antoni A e Antoni B. Podem ainda ocorrer áreas de intensidade de sinal muito alto no interior da massa, correspondendo a zonas de degeneração cística $^{(1,5,7)}$.

O tratamento de escolha é a ressecção cirúrgica do tumor. Por serem geralmente bem encapsulados, são facilmente ressecáveis, sendo que em alguns casos conseguese removê-los sem causar dano no nervo envolvido $^{(\mathbf{1 0})}$. Embora rara, pode ocorrer recorrência após cirurgia, devido à ressecção incompleta do tumor. A radioterapia não está indicada para o tratamento dos schwannomas, já que são tumores pouco sensíveis a essa terapia ${ }^{(\mathbf{2 , 5}, \mathbf{9})}$.

A ocorrência de transformação maligna é extremamente rara. Na grande maioria dos casos apresentam excelente prognóstico ${ }^{(7,8)}$.

Assim, a RM nos permite avaliar a localização da lesão, porém o diagnóstico definitivo só é feito com o estudo histopatológico. Dessa forma, o diagnóstico precoce por meio da RM e a exérese cirúrgica tornam-se a melhor forma de abordagem, com bom prognóstico.

\section{REFERÊNCIAS}

1. Atlas SW, Lavi E, Goldberg HI. Extraaxial brain tumors. In: Atlas SW, ed. Magnetic resonance imaging of the brain and spine. 3rd ed. Philadelphia, PA: Lippincott Williams \& Wilkins, 2002:695-772.

2. Stull MA, Moser RP Jr, Kransdorf MJ, Bogumill GP, Nelson MC. Magnetic resonance appearance of peripheral nerve sheath tumors. Skeletal Radiol 1991;20:9-14.

3. Kieffer AS, Chang J. Intracranial neoplasms. In: Haaga JR, Lanzieri CF, Gilkeson RC, eds. CT and MR imaging of the whole body. 4th ed. St. Louis, MO: Mosby, 2002:124-206.

4. Girolami U, Frosch MP, Anthony DC. The central nervous system. In: Cotran RS, Kumar V, Robbins SL, eds. Robbins Pathologic basis of disease. 5th ed. Philadelphia, PA: Saunders, 1994:1295-356.

5. Chui MC, Bird BL, Rogers J. Extracranial and extraspinal nerve sheath tumors: computed tomography evaluation. Neuroradiology 1988;30:47-53.

6. Al-Ghamdi S, Black MJ, Lafond G. Extracrania head and neck schwannomas. J Otolaryngol 1992; 21:186-8.

7. Fabrizi AP, Poppi M, Guiliani G, Gambari PI, Gaist G. Benign solitary nerve sheath tumors of the spinal accessory nerve in the posterior triangle of the neck. Report of two cases. J Neurosurg Sci 1992; 36:247-50.

8. Suh JS, Abenoza P, Galloway HR, Everson LI, Griffiths HJ. Peripheral (extracranial) nerve tumors: correlation of MR imaging and histologic findings. Radiology 1992;183:341-6.

9. Embry BM, Pritchard R. Trigeminal neurilemmoma: appearance on magnetic resonance imaging. South Med J 1989;82:653-6.

10. Lunardi P, Missori P, Gagliardi FM, Fraioli B. Trigeminal schwannoma with infratemporal extension Case report. J Neurosurg Sci 1989;33:293-5.

11. Osborn AG. Classificação e diagnóstico diferencial. In: Osborn AG, ed. Diagnóstico neurorradiológico. Rio de Janeiro, RJ: Revinter, 1999:401-528. 